Rev. Int. Contam. Ambie. 34 (3) 405-416, 2018

DOI: 10.20937/RICA.2018.34.03.04

\title{
CEPAS MICROBIANAS CON POTENCIAL PARA SUSTITUIR LA FERTILIZACIÓN INORGÁNICA DE SORGO DULCE (Sorghum bicolor)
}

\author{
Arturo DÍAZ-FRANCO ${ }^{1}$, Armando AGUADO-SANTACRUZ ${ }^{2}$, Raymundo ROSAS-QUIJANO ${ }^{3}$, \\ Alfredo VÁZQUEZ-OVANDO ${ }^{3}$ y Didiana GÁLVEZ-LÓPEZ ${ }^{3 *}$
}

${ }^{1}$ INIFAP, Campo Experimental Río Bravo, carretera Matamoros-Reynosa km 61, 88900 Río Bravo, Tamaulipas, México

${ }^{2}$ INIFAP, Campo Experimental Bajío, carretera Celaya-San Miguel de Allende, 38010 Celaya, Guanajuato, México

${ }^{3}$ Instituto de Biociencias, Universidad Autónoma de Chiapas, Boulevard Príncipe Akishino s/n. Col. Solidaridad 2000, Tapachula, Chiapas, México, CP 30798

*Autor para correspondencia: didiana.galvez@unach.mx

(Recibido mayo 2017; aceptado octubre 2017)

Palabras clave: biofertilización, características de planta, productividad, hongos micorrízico-arbusculares, bacterias promotoras del crecimiento vegetal, sorgo dulce, sostenibilidad

\section{RESUMEN}

El sorgo dulce (Sorghum bicolor) es un cultivo destinado a la producción de azúcar refinada, alcohol y biocombustibles, entre otros. El uso de fertilizantes de síntesis química, aunque es necesario, representa una práctica de alto costo económico y ambiental. Los hongos micorrízicos arbusculares y las bacterias promotoras de crecimiento vegetal empleadas como biofertilizantes, tienen la capacidad de reducir e incluso sustituir a la fertilización inorgánica. El objetivo del presente trabajo fue evaluar el potencial biofertilizante de cepas microbianas mediante ensayos en invernadero con la Variedad Dale, y la productividad en campo con las variedades Dale y Cañero, comparado con la fertilización convencional. Se observó una respuesta simbiótica favorable de las cepas microbianas en invernadero y campo, en ambas variedades de sorgo dulce. En general, en invernadero las cepas microbianas promovieron mejores características de planta (índice de clorofila, altura, diámetro de tallo y contenido de azúcares) y biomasa (tallo, hojas, inflorescencia, aérea y radicular), respecto a plantas no inoculadas. La respuesta en campo de las variedades al efecto de las cepas microbianas fue competitiva respecto a la fertilización convencional. La biomasa de tallo y el contenido de azúcares, que son las principales variables de productividad del sorgo dulce, fueron similares entre las cepas microbianas y la fertilización inorgánica convencional. Los resultados obtenidos demostraron que las cepas evaluadas tienen potencial para sustituir a la fertilización inorgánica.

Key words: biofertilization, plant characteristics, productivity, arbuscular mycorrhizal fungi, plant growth promoting bacteria, sweet sorghum, sustainability 


\begin{abstract}
Sweet sorghum (Sorghum bicolor) is a crop destined for the production of refined sugar, alcohol, and biofuels, among others. Inorganic fertilization, although necessary, represents a practice of high economic and environmental cost. Arbuscular mycorrhizal fungi and plant growth promoting bacteria used as biofertilizers have the capacity to reduce or even replace inorganic fertilization. The objective of the present work was to evaluate the biofertilizer potential of microbial strains by greenhouse tests with variety Dale, and productivity in the field with varieties. Dale and Cañero, compared to conventional fertilization. A favorable symbiotic response of microbial strains was observed in greenhouse, field and in both varieties of sweet sorghum. In greenhouse, in general, the microbial strains promoted better plant characteristics (chlorophyll index, height, stem diameter and sugars content) and biomass (stem, leaves, inflorescence, aerial and radicle), compared to uninoculated plants. At field scale the response of varieties to the effect of microbial strains was competitive in relation to conventional fertilization. Stem biomass and sugar content, main productivity variables of sweet sorghum, were similar between microbial strains and conventional inorganic fertilization. The results obtained showed that the strains evaluated have the potential to substitute inorganic fertilization.
\end{abstract}

\section{INTRODUCCIÓN}

El sorgo dulce (Sorghum bicolor) es un cultivo que no compite con aquellos destinados a la alimentación, puede sembrarse en áreas susceptibles de sequía y otras condiciones adversas de producción, y posee amplia adaptación, rápido crecimiento, producción de biomasa y alta acumulación de azúcares, en especial en el tallo (Calviño y Messing 2012, Rolz et al. 2017). Sus usos son variados, desde la producción de azúcar refinada y alcohol, hasta biocombustibles y otros productos (Regassa y Wortmann 2014). Los requerimientos de fertilización y las dosis han sido ampliamente documentadas en esta gramínea (Rego et al. 2003, Montes et al. 2010, Mau et al. 2016). En México, para la región norte del estado de Tamaulipas, la dosis de fertilización recomendada para sorgo dulce es de 120 y $40 \mathrm{~kg} / \mathrm{ha}$ de N y P, respectivamente (Montes et al. 2010). Sin embargo, esta práctica tiene repercusiones económicas (implica el uso de insumos de alto costo) y ambientales importantes.

La reducción en la cantidad de recursos energéticos no renovables ha provocado en los últimos años incremento en los precios de los combustibles, fertilizantes y otros insumos derivados. Aunado a esto, el calentamiento global debido a actividades humanas y al uso de fuentes de energía que liberan gases de efecto invernadero (como el $\mathrm{CO}_{2}$ ), es uno de los grandes problemas que enfrenta la sociedad contemporánea. Por ello resulta importante iniciar programas alternativos encaminados a contar con una producción agrícola sustentable, como los biocombustibles que producen energía limpia y eficiente. Este manejo alterno de producción de energía puede coadyuvar a reducir la contaminación y los gases de efecto invernadero que afectan el clima (Prasad et al. 2007, Almodares y Hadi 2009), así como a reducir o sustituir la cantidad de fertilizantes inorgánicos aplicadas en los cultivos, las cuales generan contaminación de los agroecosistemas, eutrofización de mantos acuíferos y otros efectos indeseables (Adesemoye et al. 2009, Berruti et al. 2016).

La biofertilización basada en el empleo de microorganismos benéficos representa una importante alternativa tecnológica, amigable con el ambiente y con potencial para coadyuvar en la producción agrícola, particularmente en la promoción de la productividad. Dada la necesidad del manejo sostenible de los sistemas agrícolas, resulta crucial el uso de microorganismos benéficos para la conservación y fertilidad de los suelos (Hamel y Desire 2006, Adesemoye y Kloepper 2009, Nadeem et al. 2014). En este sentido, reviste gran importancia la preparación de inoculantes que tengan efectividad sobre las plantas, en especial aquellas de importancia económica y social. Entre los microorganismos benéficos, los hongos micorrízicos arbusculares (HMA) y las bacterias promotoras de crecimiento vegetal (BPCV) son los microorganismos más estudiados (Nadeem et al. 2014). Dentro de la actividad simbiótica, los HMA manifiestan diferentes mecanismos que inducen mayor exploración del suelo a través del crecimiento de las hifas; disminuyen los efectos de condiciones abióticas adversas para 
la planta; producen fitohormonas que estimulan el crecimiento de la planta; facilitan la absorción de nutrimentos; producen proteínas como la glomalina, que promueve la adhesión de las partículas del suelo favoreciendo la agregación, e inducen una acción protectora contra algunos fitopatógenos del suelo (Smith y Read 2008, Berruti et al. 2016). El grupo de BPCV puede estimular el crecimiento de las plantas a través de producción de fitohormonas, fijación de $\mathrm{N}$ o biocontrol de fitopatógenos mediante compuestos antifúngicos como sideróforos o enzimas líticas (Vessey 2003, Sharma et al. 2012).

Varios estudios se han enfocado a conocer el efecto de los microorganismos del suelo como biofertilizantes cuando se les utiliza para incrementar la producción (Hungria et al. 2010, Alvarado et al. 2014), sustituir o disminuir la fertilización inorgánica (Amaya et al. 2005, Adesemoye et al. 2009), conferir tolerancia contra patógenos del suelo (Jetiyanon et al. 2003), contribuir a la biorremediación de suelos contaminados (Franco et al. 2007) y proveer tolerancia a otros factores abióticos (Díaz et al. 2011, Nadeem et al. 2014). No obstante, algunas cepas de microorganismos benefician a un determinado hospedero en contraposición a otros, además de que su funcionalidad puede alterarse en determinadas condiciones edafoclimáticas, hecho que muestra las marcadas diferencias existentes entre especies e incluso entre cepas de la misma especie (Klironomos 2003, Rodríguez et al. 2004, Hungria et al. 2010, Montero et al. 2010). Varios inoculantes se preparan a partir de cepas microbianas introducidas o extranjeras, aunque actualmente se ha dado énfasis a la utilización de cepas nativas, que puedan ser reintroducidas a través de su inoculación a cultivos, y tienen mayor capacidad de adaptación y efectividad en sitios y climas específicos (Plenchette et al. 2005, Hungria et al. 2010, Tchabi et al. 2010).

Hay diversos microorganismos con características simbióticas como las BPCV y HMA, que han mostrado la importancia de las interacciones entre las asociaciones planta-microorganismo con la capacidad de obtener beneficios agronómicos eficientes, incluso como sustitutos de la fertilización inorgánica (Wani y Lee 2002, Sharma et al. 2012, Orona et al. 2013). No obstante, es poco conocida la influencia de la biofertilización en la cantidad producida de biomasa y azúcares en el sorgo dulce. En un estudio previo, Díaz et al. (2016) concluyeron que la cantidad de biomasa y azúcares en el tallo del sorgo dulce fueron semejantes cuando se aplicó fertilización inorgánica convencional y cuando se utilizó inoculación de $R$. intraradices combinada con el $50 \%$ de la fertilización inorgánica. Por lo anterior, el objetivo del estudio fue comparar la productividad del sorgo dulce con inoculación de cepas microbianas y fertilización convencional.

\section{MATERIALES Y MÉTODOS}

\section{Experimento en invernadero}

El estudio se desarrolló en el invernadero del Campo Experimental Río Bravo (CERIB) del Instituto Nacional de Investigaciones Forestales Agrícolas y Pecuarias (INIFAP), Río Bravo, Tamaulipas ( $\left.25^{\circ} 57^{\prime} \mathrm{N}, 98^{\circ} 01^{\prime} \mathrm{O}, 25 \mathrm{msnm}\right)$. Como sustrato se empleó la mezcla de suelo Termolita ${ }^{\circledR}(90: 10 \mathrm{v} / \mathrm{v})$ y se esterilizó con bromuro de metilo. Bolsas de plástico de $9 \mathrm{~kg}$ de capacidad se llenaron con el sustrato. Las características del suelo fueron: materia orgánica (MO), $1.4 \% ; \mathrm{pH}, 8.0$; conductividad eléctrica (CE), $1.1 \mathrm{dS} / \mathrm{m}$; N, P, K, 13.7, 22 y $907 \mathrm{mg} / \mathrm{kg}$, respectivamente, con textura franco-arenosa. El suelo, que se obtuvo de un muestreo a profundidad $0-30 \mathrm{~cm}$ de acuerdo con la cartografía del Instituto Nacional de Estadística y Geografía (INEGI), corresponde al tipo vertisol según clasificación de la Organización de las Naciones Unidas para la Alimentación y la Agricultura (INEGI 2017). El pH se determinó en solución acuosa (1:2), la CE mediante conductímetro con pasta de saturación, la MO se analizó mediante el método de dicromato de potasio (Walkely y Black), el nitrógeno inorgánico $\left(\mathrm{NO}_{3}-\mathrm{N}\right)$ se determinó mediante ácido acetilsalicílico, el $\mathrm{P}$ disponible se midió con el método de Olsen y el K intercambiable con acetato de amonio (Plenecassagne et al. 1999).

\section{Cepas microbianas}

Se utilizaron cepas microbianas del Instituto Nacional de Investigaciones INIFAP. Se evaluaron tres cepas de HMA: M35 (Glomus mossae), M20 (Gigaspora albida) у M3 (G. mossae), proporcionadas por el Campo Experimental General Terán del INIFAP. Las BPCV empleadas fueron cuatro: Pseudomonas sp. (BPSON), Herbaspirillum sp. (BH), Ranhella aquatilis (BRA) y Azospirillum sp. (BAZO), facilitadas por el Campo Experimental Bajío del INIFAP. El inóculo de los HMA fue de $\geq 60$ esporas/g de suelo y las rizobacterias tuvieron una concentración de $10^{7}-10^{8}$ unidades formadoras de colonias (UFC)/g de turba. Cada uno de los microrganismos fue evaluado como tratamiento de manera individual. Como testigos se emplearon la inoculación de micorriza INIFAP ${ }^{\circledR} \mathrm{M}$ (Rhizophagus intraradices) y un absoluto sin inoculación. 


\section{Manejo experimental}

La inoculación del sustrato con los microrganismos al suelo fue a razón de $2 \mathrm{~g}$ de turba (inoculo bacteriano) y $4 \mathrm{~g}$ de suelo (inoculo micorrízico) por bolsa, a una profundidad de $10-11 \mathrm{~cm}$. El inóculo se cubrió con una capa de suelo de $\approx 5 \mathrm{~cm}$. La siembra se realizó el 17 de febrero de 2012. Se desinfectaron las semillas de la variedad de sorgo dulce Dale mediante una solución de hipoclorito de sodio al $10 \%$ por 10 min y se sembraron cinco semillas en cada bolsa para después dejar una plántula. Se empleó un diseño completamente aleatorio con 10 repeticiones por tratamiento. Las plantas se regaron de forma regular y se mantuvieron durante tres meses hasta el estado de grano lechoso-masoso (15 de mayo). En el estado de floración, se determinó el índice de clorofila foliar en la parte superior de la planta en unidades SPAD, obtenidas con un medidor Minolta SPAD-502. Las variables evaluadas en la etapa de grano lechosomasoso fueron: altura de planta; diámetro de tallo (de la parte intermedia); biomasa fresca (g/planta) de tallo, hojas, inflorescencia y raíz, y contenido de azúcares solubles totales (grados Brix) mediante refractómetro, obtenido del promedio de lecturas en cada dos entrenudos del tallo $(2,4,6)$ (Díaz et al. 2016). Los datos se procesaron mediante análisis de varianza y las diferencias entre medias se detectaron con la prueba de Tukey $(\mathrm{p} \leq 0.05)$. Además, se hicieron correlaciones entre variables mediante análisis de correlación de Pearson $(\mathrm{p} \leq 0.05)$.

\section{Evaluación en campo}

En los terrenos del Campo Experimental Río Bravo se establecieron los experimentos el 27 de febrero de 2012, en un suelo de textura franco-limosa, diferente al utilizado en invernadero, con las siguientes características de presiembra: MO, $1.28 \% ; \mathrm{pH}, 8.6$; CE, $1.26 \mathrm{ds} / \mathrm{m}$; N, $5.7 \mathrm{mg} / \mathrm{kg}$; P, $13.3 \mathrm{mg} / \mathrm{kg}$, y K, $1224 \mathrm{mg} / \mathrm{kg}$.

\section{Manejo experimental}

Se utilizaron las variedades comerciales de sorgo dulce Cañero y Dale, las cuales se sembraron en experimentos independientes. En cada variedad se evaluaron como inoculantes microbianos cuatro cepas de HMA: M35 (Glomus mossae), M20 (Gigaspora albida), M3 (G. mossae) y micorriza INIFAP $^{\circledR} \mathrm{M}$ (Rhizophagus intraradices), así como tres cepas de BPCV: BPSON, BAZO y BH. Los testigos consistieron en fertilización convencional con 120 y $40 \mathrm{~kg} / \mathrm{ha}$ de N y P (urea y fosfato monoamónico), respectivamente (Montes et al. 2010), así como un testigo absoluto. Los nueve tratamientos se distribuyeron en un diseño de bloques al azar repetidos cuatro veces en parcelas de tres surcos de 5 m de longitud $\left(12.3 \mathrm{~m}^{2}\right)$. La inoculación microbiana se hizo en la semilla $(6 \mathrm{~kg} / \mathrm{ha})$ y las dosis consistieron en $500 \mathrm{y}$ $200 \mathrm{~g} / \mathrm{ha}$ para los HMA y las BPCV, respectivamente. Respecto al fertilizante inorgánico, al momento de la siembra se aplicó la mitad de $\mathrm{N}$ y todo el P. El N restante se aplicó 45 días después de la siembra. La densidad de plantas se ajustó a 145000 plantas/ha. Para otras prácticas agronómicas se siguieron las indicaciones locales (Montes et al. 2010).

\section{Variables evaluadas}

En 10 plantas del surco central de cada parcela se midieron las siguientes variables: índice de clorofila en estado de floración con lecturas $(n=30)$ realizadas en el centro de las hojas y el tercio superior de las plantas; diámetro de tallo de la parte intermedia, y biomasa fresca de hojas, tallo, inflorescencia y raíz en estado de grano lechoso-masoso. Además, se midió con refractómetro el contenido de azúcares (reportado como grados Brix) en cada dos entrenudos del tallo (Díaz et al. 2016). Los datos se sometieron a análisis de varianza independientes y combinado (ambas variedades), con separación de medias por prueba de Tukey $(p \leq 0.05)$. Estos análisis se realizaron a través del programa SAS v. 8.1.

\section{RESULTADOS}

\section{Resultados en invernadero}

Los resultados indicaron que en todas las variables medidas las cepas microbianas inoculadas tuvieron un efecto benéfico, y que en la mayoría de los casos superaron los valores de las plantas testigo. En cuanto a características de planta, con la cepa BPSON se alcanzó mayor altura y acumulación de clorofila, con $152.4 \mathrm{~cm}$ y 40.5 unidades SPAD de índice de clorofila, respectivamente; el mayor diámetro de tallo se registró con M35, M20, M3, BPSON y M. Con relación al contenido de azúcares en tallo, la mayoría de las cepas superaron al testigo $(\mathrm{p}=0.001)($ Cuadro I).

En cuanto a las características de biomasa del sorgo dulce, en todas las variables se apreció una influencia significativa de las cepas inoculadas (Cuadro II). La cepa BPSON destacó para la biomasa de tallo con $62.7 \mathrm{~g}$. Respecto de la promoción de hojas, los mejores resultados se lograron con la cepa micorrízica M3, con $52.5 \mathrm{~g}$, y las cepas M35 y BPSON registraron los mejores promedios en cuanto a biomasas de inflorescencia y aérea total, con 30.3 y $137.5 \mathrm{~g}$, respectivamente. La biomasa aérea total se 
CUADRO I. INFLUENCIA DE CEPAS MICROBIANAS EN LAS CARACTERÍSTICAS DE PLANTAS DE SORGO DULCE CULTIVADAS EN INVERNADERO

\begin{tabular}{lcccc}
\hline \multirow{4}{*}{ Tratamiento } & \multicolumn{4}{c}{ Características de planta } \\
\cline { 2 - 5 } & $\begin{array}{c}\text { Clorofila } \\
\text { (unidades SPAD) }\end{array}$ & $\begin{array}{c}\text { Altura } \\
(\mathrm{cm})\end{array}$ & $\begin{array}{c}\text { Diámetro de } \\
\text { tallo }(\mathrm{mm})\end{array}$ & $\begin{array}{c}\text { Contenido de azúcares } \\
\text { del tallo }\left({ }^{\circ} \text { Brix) }\right.\end{array}$ \\
\hline M35 & $39.7 \mathrm{ab} *$ & $147.5 \mathrm{abc}$ & $12.3 \mathrm{a}$ & $9.1 \mathrm{ab}$ \\
M20 & $39.6 \mathrm{ab}$ & $147.0 \mathrm{abc}$ & $12.7 \mathrm{a}$ & $9.3 \mathrm{ab}$ \\
M3 & $39.4 \mathrm{ab}$ & $150.7 \mathrm{ab}$ & $12.3 \mathrm{a}$ & $9.4 \mathrm{ab}$ \\
BPSON & $40.5 \mathrm{a}$ & $152.4 \mathrm{a}$ & $12.5 \mathrm{a}$ & $9.7 \mathrm{a}$ \\
BH & $37.7 \mathrm{abc}$ & $134.9 \mathrm{cde}$ & $11.5 \mathrm{~b}$ & $9.6 \mathrm{a}$ \\
BRA & $39.5 \mathrm{ab}$ & $136.3 \mathrm{cde}$ & $10.8 \mathrm{c}$ & $9.7 \mathrm{a}$ \\
BAZO & $34.0 \mathrm{~d}$ & $137.6 \mathrm{bcd}$ & $10.8 \mathrm{c}$ & $10.1 \mathrm{a}$ \\
M & $38.2 \mathrm{abc}$ & $129.0 \mathrm{de}$ & $12.3 \mathrm{a}$ & $9.7 \mathrm{a}$ \\
Testigo & $36.6 \mathrm{~cd}$ & $123.9 \mathrm{e}$ & $10.7 \mathrm{c}$ & $7.6 \mathrm{~b}$ \\
F & 0.008 & 0.001 & 0.001 & 0.001 \\
\hline
\end{tabular}

*Letras iguales indican pertenencia al mismo grupo de promedios para cada variable $(\mathrm{p}<0.05)$ M35 (Glomus mossae), M20 (Gigaspora albida), M3 (G. mossae), BPSON (Pseudomonas sp.), BH (Herbaspirillum sp.), BRA (Ranhella aquatilis), BAZO (Azospirillum sp.), M (Rhizophagus intraradices)

CUADRO II. INFLUENCIA DE CEPAS MICROBIANAS EN LA BIOMASA DE PLANTAS DE SORGO DULCE CULTIVADAS EN INVERNADERO

\begin{tabular}{lccccc}
\hline \multirow{2}{*}{ Tratamiento } & \multicolumn{5}{c}{ Biomasa $(\mathrm{g})$} \\
\cline { 2 - 6 } & Tallo & Hojas & Inflorescencia & Aérea total & Radicular \\
\hline M35 & $53.9 \mathrm{bc} *$ & $51.3 \mathrm{abc}$ & $30.3 \mathrm{a}$ & $137.5 \mathrm{a}$ & $23.6 \mathrm{a}$ \\
M20 & $61.3 \mathrm{ab}$ & $50.3 \mathrm{abc}$ & $23.0 \mathrm{bcd}$ & $134.5 \mathrm{abc}$ & $26.3 \mathrm{a}$ \\
M3 & $61.4 \mathrm{ab}$ & $52.5 \mathrm{a}$ & $20.2 \mathrm{de}$ & $133.1 \mathrm{abcd}$ & $26.0 \mathrm{a}$ \\
BPSON & $62.7 \mathrm{a}$ & $49.1 \mathrm{abcd}$ & $27.3 \mathrm{a}$ & $139.0 \mathrm{a}$ & $26.6 \mathrm{a}$ \\
BH & $46.3 \mathrm{~cd}$ & $42.7 \mathrm{f}$ & $15.8 \mathrm{ef}$ & $104.7 \mathrm{e}$ & $16.2 \mathrm{~b}$ \\
BRA & $47.0 \mathrm{~cd}$ & $47.1 \mathrm{bcde}$ & $17.1 \mathrm{def}$ & $111.1 \mathrm{de}$ & $13.4 \mathrm{~b}$ \\
BAZO & $41.7 \mathrm{~d}$ & $39.5 \mathrm{f}$ & $13.4 \mathrm{f}$ & $94.6 \mathrm{e}$ & $13.2 \mathrm{~b}$ \\
M & $49.3 \mathrm{~cd}$ & $51.0 \mathrm{abc}$ & $13.7 \mathrm{f}$ & $114.0 \mathrm{cde}$ & $22.6 \mathrm{a}$ \\
Testigo & $40.2 \mathrm{~d}$ & $43.0 \mathrm{f}$ & $12.4 \mathrm{f}$ & $95.6 \mathrm{e}$ & $13.3 \mathrm{~b}$ \\
F & 0.001 & 0.002 & 0.005 & 0.001 & 0.001 \\
\hline
\end{tabular}

* Letras iguales indican pertenencia al mismo grupo de promedios para cada variable $(\mathrm{p}<0.05)$ M35 (Glomus mossae), M20 (Gigaspora albida), M3 (G. mossae), BPSON (Pseudomonas sp.), BH (Herbaspirillum sp.), BRA (Ranhella aquatilis), BAZO (Azospirillum sp.), M (Rhizophagus intraradices)

asoció de manera positiva con la biomasa de tallo, hojas e inflorescencia $(r=0.74-0.95)$. La mayor biomasa radicular se registró con todas las cepas de HMA, así como BPSON. Esta característica podría reflejar una mayor capacidad de absorción de nutrientes, lo que se convierte en el factor principal para el crecimiento de la planta. La asociación entre biomasa radicular $\mathrm{y}$ aérea total fue significativa $(\mathrm{p}<0.05 ; \mathrm{r}=0.83)$.

\section{Experimento de campo}

Respecto de las características de planta del sorgo dulce Cañero, las cepas microbianas tuvieron un impacto significativo en el índice de clorofila $(\mathrm{p}=0.04)$ y en el contenido de azúcares del tallo $(p=0.01)$, y no se observaron diferencias significativas para la altura de planta $(\mathrm{p}=0.41)$ ni el diámetro de tallo $(\mathrm{p}=0.42)$. Los mayores valores de índice de clorofila se obtuvieron en las parcelas inoculadas con micorriza INIFAP (M) y aquellas en las que se utilizó fertilización inorgánica convencional. En cuanto a contenido de azúcares, destacaron BPSON, BAZO, $\mathrm{BH}$ y la fertilización convencional (Cuadro III).

En las variables de biomasa se observaron efectos significativos entre tratamientos para hojas 
CUADRO III. INFLUENCIA DE CEPAS MICROBIANAS EN LAS CARACTERÍSTICAS DE PLANTAS DE SORGO DULCE VARIEDAD CAÑERO CULTIVADAS EN CAMPO

\begin{tabular}{lcccc}
\hline Tratamiento & \multicolumn{4}{c}{ Características de planta } \\
\cline { 2 - 5 } & $\begin{array}{c}\text { Clorofila } \\
\text { (unidades SPAD) }\end{array}$ & $\begin{array}{c}\text { Altura } \\
(\mathrm{m})\end{array}$ & $\begin{array}{c}\text { Diámetro del } \\
\text { tallo }(\mathrm{mm})\end{array}$ & $\begin{array}{c}\text { Contenido de azúcares } \\
\text { del tallo ('Brix) }\end{array}$ \\
\hline M35 & $35.5 \mathrm{ab}^{*}$ & 3.35 & 19.9 & $19.2 \mathrm{ab}$ \\
M20 & $34.1 \mathrm{~b}$ & 3.45 & 20.0 & $19.1 \mathrm{ab}$ \\
M3 & $33.4 \mathrm{~b}$ & 3.41 & 19.1 & $19.1 \mathrm{ab}$ \\
BPSON & $32.9 \mathrm{~b}$ & 3.29 & 18.3 & $19.7 \mathrm{a}$ \\
BAZO & $33.9 \mathrm{~b}$ & 3.35 & 18.7 & $19.7 \mathrm{a}$ \\
BH & $33.2 \mathrm{~b}$ & 3.33 & 18.3 & $19.9 \mathrm{a}$ \\
M & $36.1 \mathrm{a}$ & 3.30 & 18.4 & $19.4 \mathrm{ab}$ \\
120-40-00 & $36.6 \mathrm{a}$ & 3.54 & 19.4 & $19.7 \mathrm{a}$ \\
Testigo & $32.1 \mathrm{~b}$ & 3.28 & 18.0 & $17.7 \mathrm{~b}$ \\
F & 0.04 & 0.41 & 0.42 & 0.01 \\
\hline
\end{tabular}

* Letras iguales indican pertenencia al mismo grupo de promedios para cada variable $(\mathrm{p}<0.05)$

M35 (Glomus mossae), M20 (Gigaspora albida), M3 (G. mossae), BPSON (Pseudomonas sp.), BAZO (Azospirillum sp.), BH (Herbaspirillum sp.), M (Rhizophagus intraradices)

$(\mathrm{p}=0.031)$ y biomasa aérea total $(\mathrm{p}=0.01)$. Los tratamientos con las cepas M3 y BAZO registraron la mayor biomasa de hojas, ambas con promedio de $178.5 \mathrm{~g}$. Con relación a la biomasa aérea total todos los tratamientos superaron, de forma similar, al testigo absoluto (Cuadro IV).

Los tratamientos influyeron significativamente en el índice de clorofila $(\mathrm{p}=0.02)$ y en el contenido de azúcares en el tallo $(p=0.004)$ de la variedad Dale. Los mayores valores de contenido de clorofila se observaron en las parcelas con la cepa M3 y la fertilización inorgánica, mientras que la cepa M35 destacó en contenido de azúcar. Al igual que en la variedad Cañero, los tratamientos no tuvieron efectos significativos en altura de planta y diámetro de tallo (Cuadro V). Contrario a la respuesta obtenida

CUADRO IV. INFLUENCIA DE CEPAS MICROBIANAS EN LA BIOMASA DE PLANTAS DE SORGO DULCE VARIEDAD CAÑERO CULTIVADAS EN CAMPO

\begin{tabular}{lccccc}
\hline Tratamiento & \multicolumn{5}{c}{ Biomasa $(\mathrm{g})$} \\
\cline { 2 - 6 } & Tallo & Hojas & Inflorescencia & Aérea total & Radicular \\
\hline M35 & 576 & $157 \mathrm{ab}^{*}$ & 73 & $806 \mathrm{a}$ & 81 \\
M20 & 523 & $149 \mathrm{ab}$ & 68 & $740 \mathrm{a}$ & 71 \\
M3 & 572 & $176 \mathrm{a}$ & 71 & $819 \mathrm{a}$ & 77 \\
BPSON & 502 & $156 \mathrm{ab}$ & 69 & $737 \mathrm{a}$ & 80 \\
BAZO & 594 & $181 \mathrm{a}$ & 75 & $850 \mathrm{a}$ & 88 \\
BH & 493 & $154 \mathrm{ab}$ & 74 & $739 \mathrm{a}$ & 74 \\
M & 520 & $148 \mathrm{ab}$ & 75 & $745 \mathrm{a}$ & 71 \\
120-40-00 & 536 & $148 \mathrm{ab}$ & 80 & $759 \mathrm{a}$ & 74 \\
Testigo & 457 & $131 \mathrm{~b}$ & 68 & $656 \mathrm{~b}$ & 68 \\
F & 0.51 & 0.031 & 0.67 & 0.01 & 0.71 \\
\hline
\end{tabular}

* Letras iguales indican pertenencia al mismo grupo de promedios para cada variable (Tukey, $\mathrm{p}<0.05$ )

M35 (Glomus mossae), M20 (Gigaspora albida), M3 (G. mossae), BPSON (Pseudomonas sp.), BAZO (Azospirillum sp.), BH (Herbaspirillum sp.), M (Rhizophagus intraradices) 
CUADRO V. INFLUENCIA DE CEPAS MICROBIANAS EN LAS CARACTERÍSTICAS DE PLANTAS DE SORGO DULCE VARIEDAD DALE CULTIVADAS EN CAMPO

\begin{tabular}{lcccc}
\hline Tratamiento & \multicolumn{4}{c}{ Características de planta } \\
\cline { 2 - 5 } & $\begin{array}{c}\text { Clorofila } \\
\text { (unidades SPAD) }\end{array}$ & $\begin{array}{c}\text { Altura } \\
(\mathrm{m})\end{array}$ & $\begin{array}{c}\text { Diámetro del } \\
\text { tallo }(\mathrm{mm})\end{array}$ & $\begin{array}{c}\text { Contenido de azúcares } \\
\text { del tallo ('Brix) }\end{array}$ \\
\hline M35 & $31.8 \mathrm{c} *$ & 3.08 & 20.0 & $21.3 \mathrm{a}$ \\
M20 & $34.8 \mathrm{abc}$ & 3.10 & 19.6 & $20.6 \mathrm{bc}$ \\
M3 & $37.4 \mathrm{a}$ & 3.07 & 19.0 & $21.2 \mathrm{ab}$ \\
BPSON & $35.2 \mathrm{ab}$ & 3.07 & 19.3 & $21.2 \mathrm{ab}$ \\
BAZO & $34.8 \mathrm{abc}$ & 3.09 & 20.3 & $20.8 \mathrm{abc}$ \\
BH & $33.1 \mathrm{bc}$ & 3.10 & 20.6 & $20.1 \mathrm{c}$ \\
M & $35.3 \mathrm{abc}$ & 3.05 & 19.3 & $20.9 \mathrm{abc}$ \\
120-40-00 & $37.3 \mathrm{a}$ & 3.12 & 19.4 & $20.9 \mathrm{abc}$ \\
Testigo & $32.4 \mathrm{c}$ & 3.07 & 18.4 & $18.1 \mathrm{~d}$ \\
F & 0.02 & 0.74 & 0.52 & 0.004 \\
\hline
\end{tabular}

* Letras iguales indican pertenencia al mismo grupo de promedios para cada variable (Tukey, $\mathrm{p}<0.05$ )

M35 (Glomus mossae), M20 (Gigaspora albida), M3 (G. mossae), BPSON (Pseudomonas sp.), BAZO (Azospirillum sp.), BH (Herbaspirillum sp.), M (Rhizophagus intraradices)

con la variedad Cañero, para Dale no se registraron diferencias significativas entre los tratamientos para las características de biomasa de planta (Cuadro VI).

El análisis combinado de las dos variedades demostró que las cepas microbianas incidieron en los valores de índice de clorofila $(\mathrm{p}=0.03)$ y en la cantidad de azúcares en el tallo $(p=0.02)$. Entre las variedades, Dale sólo destaca como la mayor productora de contenido de azúcares, con $20.8{ }^{\circ}$ Brix. En el resto de las variables, las variedades mostraron respuesta semejante. No se observaron interacciones significativas entre los dos factores (Cuadro VII).

Destacó que el mayor índice de clorofila se registró en las parcelas que llevaron la fertilización inorgánica (37.0 unidades SPAD). En el contenido de azúcares del tallo, tanto las cepas microbianas como la fertilización inorgánica, superaron de manera semejante, al testigo absoluto. En el resto de las características de planta, los tratamientos no mostraron influencia (Cuadro VII).

CUADRO VI. INFLUENCIA DE CEPAS MICROBIANAS EN LA BIOMASA DE PLANTAS DE SORGO DULCE VARIEDAD DALE CULTIVADAS EN CAMPO

\begin{tabular}{lccccc}
\hline \multirow{2}{*}{ Tratamiento } & \multicolumn{5}{c}{ Biomasa $(\mathrm{g})$} \\
\cline { 2 - 6 } & Tallo & Hojas & Inflorescencia & Aérea total & Radicular \\
\hline M35 & 492 & 160 & 77 & 729 & 93 \\
M20 & 534 & 170 & 81 & 785 & 112 \\
M3 & 518 & 145 & 90 & 753 & 108 \\
BPSON & 544 & 141 & 76 & 761 & 97 \\
BAZO & 516 & 145 & 86 & 747 & 116 \\
BH & 551 & 177 & 85 & 813 & 125 \\
M & 493 & 140 & 68 & 671 & 92 \\
120-40-00 & 497 & 153 & 68 & 703 & 91 \\
Testigo & 434 & 141 & 68 & 652 & 83 \\
F & 0.18 & 0.52 & 0.14 & 0.059 & 0.18 \\
\hline
\end{tabular}

M35 (Glomus mossae), M20 (Gigaspora albida), M3 (G. mossae), BPSON (Pseudomonas sp.), BAZO (Azospirillum sp.), BH (Herbaspirillum sp.), M (Rhizophagus intraradices) 
CUADRO VII. INFLUENCIA DE CEPAS MICROBIANAS EN LAS CARACTERÍSTICAS DE PLANTAS DE SORGO DULCE VARIEDADES CAÑERO Y DALE CULTIVADAS EN CAMPO

\begin{tabular}{lcccc}
\hline Factor & \multicolumn{4}{c}{ Características de planta } \\
\cline { 2 - 5 } & $\begin{array}{c}\text { Clorofila } \\
\text { (unidades SPAD) }\end{array}$ & $\begin{array}{c}\text { Altura } \\
(\mathrm{m})\end{array}$ & $\begin{array}{c}\text { Diámetro } \\
(\mathrm{mm})\end{array}$ & $\begin{array}{c}\text { Tallo } \\
\left({ }^{\circ} \text { Brix }\right)\end{array}$ \\
\hline \multicolumn{5}{c}{ Cepa } \\
M35 & $33.7 \mathrm{bc} *$ & 3.22 & 19.9 & $20.2 \mathrm{a}$ \\
M20 & $34.5 \mathrm{bc}$ & 3.27 & 19.8 & $19.8 \mathrm{a}$ \\
M3 & $35.4 \mathrm{abc}$ & 3.24 & 19.0 & $20.1 \mathrm{a}$ \\
BPSON & $34.4 \mathrm{bc}$ & 3.18 & 18.8 & $20.4 \mathrm{a}$ \\
BAZO & $34.4 \mathrm{bc}$ & 3.22 & 19.4 & $20.2 \mathrm{a}$ \\
BH & $33.1 \mathrm{c}$ & 3.22 & 19.4 & $20.1 \mathrm{a}$ \\
M & $35.7 \mathrm{ab}$ & 3.17 & 18.8 & $20.1 \mathrm{a}$ \\
120-40-00 & $37.0 \mathrm{a}$ & 3.28 & 19.3 & $20.2 \mathrm{a}$ \\
Testigo & $33.0 \mathrm{c}$ & 3.18 & 18.2 & $17.9 \mathrm{~b}$ \\
F & 0.03 & 0.36 & 0.31 & 0.02 \\
\hline \multicolumn{5}{c}{ Variedad } \\
\hline Cañero & 34.8 & 3.36 & 18.8 & 19.3 \\
Dale & 34.4 & 3.08 & 19.5 & 20.8 \\
F & 0.5 & 0.053 & 0.051 & 0.001 \\
Cepa x Variedad & 0.1 & 0.23 & 0.57 & 0.17 \\
\hline
\end{tabular}

* Letras iguales indican pertenencia al mismo grupo de promedios para cada variable (Tukey, $\mathrm{p}<0.05$ )

M35 (Glomus mossae), M20 (Gigaspora albida), M3 (G. mossae), BPSON (Pseudomonas sp.), BAZO (Azospirillum sp.), BH (Herbaspirillum sp.), M (Rhizophagus intraradices)

En las variables de biomasa de planta se observaron diferencias significativas para la biomasa del tallo $(\mathrm{p}=0.03)$ y aérea total $(\mathrm{p}=0.04)$. Todos los tratamientos superaron al testigo con relación a la biomasa de tallo y las cepas M3 y BAZO produjeron los mayores valores para la biomasa aérea total. Entre las variedades, Dale superó a Cañero $(\mathrm{p}=0.001)$ con diferencia de $26 \mathrm{~g}$ en cuanto a biomasa radicular. En otras variables, así como en las interacciones, no se observaron diferencias significativas (Cuadro VIII). Se observó una asociación positiva entre el contenido de azúcares del tallo con la biomasa de tallo $(\mathrm{r}=0.67)$ y con la biomasa aérea total $(r=0.59)$.

\section{DISCUSIÓN}

Los resultados obtenidos en condiciones de invernadero indican que todas las cepas microbianas experimentales tuvieron efectos significativos en las características de planta y biomasa del sorgo dulce Dale, con evidente incremento respecto al testigo tanto en los azúcares y la biomasa del tallo, como en la mayoría de variables. En esta fase del estudio, destacó la cepa BPSON (Pseudomonas sp.) con el mayor potencial promotor del índice de clorofila, altura de planta y biomasa de tallo. Bécquer et al. (2012) reportaron incrementos en biomasa aérea de trigo (Triticum aestivum) cuando fue inoculado con cepas de Azospirillum zeae y Sinorhizobium meliloti en condiciones controladas. De igual forma, en maíz (Zea mays) de invernadero, Boucher et al. (1999) reportaron incrementos de 16 a $24 \%$ en la biomasa foliar con cuatro especies de HMA. Es importante destacar que la mayor biomasa radicular se registró con todas las cepas de HMA y BPSON, respuesta que puede explicarse por el efecto hormonal, ya que algunos microorganismos tienen esa capacidad. Al respecto, Ilyas y Bano (2010) y Bécquer et al. (2012) informaron mayor desarrollo radicular de plántulas de trigo mediante la inoculación de cepas microbianas, asociado con mayor producción de fitohormonas.

No obstante, la respuesta de las variedades Cañero y Dale a la inoculación de los microorganismos en campo fue diferente entre ambas y respecto de los 
CUADRO VIII. INFLUENCIA DE CEPAS MICROBIANAS EN LA BIOMASA DE PLANTAS DE SORGO DULCE VARIEDADES CAÑERO Y DALE EN CAMPO, SEMBRADAS EN EL CAMPO EXPERIMENTAL RÍO BRAVO DURANTE 2012

\begin{tabular}{lccccc}
\hline \multirow{2}{*}{ Factor } & \multicolumn{5}{c}{ Biomasa $(\mathrm{g})$} \\
\cline { 2 - 7 } & Tallo & Hojas & Inflorescencia & Aérea total & Radicular \\
\hline \multicolumn{5}{c}{ Cepa } \\
\hline M35 & $534 \mathrm{a}^{*}$ & 159 & 75 & $768 \mathrm{ab}$ & 87 \\
M20 & $528 \mathrm{a}$ & 159 & 75 & $762 \mathrm{~b}$ & 91 \\
M3 & $545 \mathrm{a}$ & 160 & 81 & $786 \mathrm{a}$ & 93 \\
BPSON & $523 \mathrm{a}$ & 148 & 73 & $744 \mathrm{~b}$ & 88 \\
BAZO & $555 \mathrm{a}$ & 163 & 80 & $798 \mathrm{a}$ & 102 \\
BH & $522 \mathrm{a}$ & 165 & 80 & $767 \mathrm{ab}$ & 100 \\
M & $506 \mathrm{a}$ & 169 & 77 & $732 \mathrm{~b}$ & 81 \\
120-40-00 & $509 \mathrm{a}$ & 148 & 74 & $731 \mathrm{~b}$ & 82 \\
Testigo & $445 \mathrm{~b}$ & 139 & 68 & $652 \mathrm{c}$ & 75 \\
F & 0.03 & 0.25 & 0.37 & 0.041 & 0.19 \\
\hline & & \multicolumn{5}{c}{ Variedad } & 735 & 76 \\
\hline Cañero & 505 & 152 & 78 & 756 & 102 \\
Dale & 528 & 155 & 73 & 0.06 & 0.001 \\
F & 0.25 & 0.64 & 0.058 & 0.12 & 0.57 \\
Cepa x variedad & 0.66 & 0.16 & 0.19 & \\
\hline
\end{tabular}

* Letras iguales indican pertenencia al mismo grupo de promedios para cada variable (Tukey, $\mathrm{p}<0.05$ )

M35 (Glomus mossae), M20 (Gigaspora albida), M3 (G. mossae), BPSON (Pseudomonas sp.), BAZO (Azospirillum sp.), BH (Herbaspirillum sp.), M (Rhizophagus intraradices)

resultados obtenidos en invernadero en suelo estéril. En la variedad Cañero, las cepas microbianas afectaron significativamente el índice de clorofila, los azúcares del tallo, la biomasa de hojas y el aérea total. En la variedad Dale los microorganismos probados influyeron en el índice de clorofila y en los azúcares del tallo. A diferencia de los efectos alcanzados en el experimento de invernadero, en campo los inoculantes microbianos no influyeron en la biomasa radicular. El análisis combinado de las dos variedades destacó que todas las cepas incrementaron de manera similar el contenido de azúcares y la biomasa del tallo, en el mismo nivel que el obtenido con la fertilización convencional. Las dos variables mencionadas son las que tienen mayor relación con la productividad del sorgo dulce. Askary et al. (2009) y Bécquer et al. (2012) indicaron que el aumento de los pigmentos fotosintéticos podría estar asociado con el incremento en el aporte de $\mathrm{N}$ a la planta por la actividad simbiótica de cepas eficientes. El rango obtenido de grados Brix para las dos variedades fue de 17.9-20.4 y se encuentra comprendido dentro del rango registrado para genotipos de sorgo dulce (13-24 ${ }^{\circ}$ Brix $)$ señalado por Regassa y Wortmann (2014). Los resultados de Muthukumarasamy et al. (2006) demostraron que el total de biomasa y $\mathrm{N}$ foliar fueron mayores en las plantas de caña de azúcar (Saccharum officinarum) inoculadas con Gluconacetobacter diazotrophicus y Herbaspirillum sp., respecto a las plantas fertilizadas con dosis recomendada de $\mathrm{N}$ inorgánico $(280 \mathrm{~kg} / \mathrm{ha})$.

Diversos factores podrían explicar las diferencias en la actividad simbiótica de las cepas en ambientes distintos (invernadero y campo) y entre las dos variedades de sorgo dulce. Wani y Lee (2002) y Sharma et al. (2012) indicaron que los inoculantes microbianos se encuentran sujetos a un rango de condiciones ambientales que pueden ser hostiles, de tal forma que la sobrevivencia y su efectividad en la planta hospedera está definida por múltiples factores, dentro de los cuales están la misma planta hospedera, las condiciones edafoclimáticas, el antagonismo, la competencia o desplazamiento por la biodiversidad microbiana nativa, y el manejo de las prácticas agronómicas. Por lo anterior, es posible que las cepas que mostraron efectividad en las condiciones de invernadero pudieran ser eficientes en otras 
condiciones ambientales. Además, es conocido que algunas cepas microbianas (HMA o BPCV), incluidas las de la misma especie, muestran especificidad y benefician en mayor grado a un determinado hospedero o genotipo en comparación con otros (Klironomos 2003, Hungria et al. 2010).

En estudios relacionados (Boucher et al. 1999, Matiru y Dakora 2004, Berruti et al. 2016) se ha enfatizado la necesidad de seleccionar HMA y BPCV eficientes para incrementar el crecimiento y la productividad de los cultivos. Resultados semejantes a los obtenidos se reportaron para caña de azúcar (Lopes et al. 2012), los cuales demostraron variaciones de efectividad de diferentes inoculantes microbianos para altura de planta, diámetro de tallo y contenido de azúcares. Los resultados obtenidos en el presente estudio indican que las cepas evaluadas pueden ser competitivas en productividad respecto a la fertilización inorgánica para el cultivo de sorgo dulce, además de que son nativas y están adaptadas a las condiciones ambientales de la región productora de sorgo en México. La producción equivalente entre inoculantes microbianos y fertilización inorgánica también se ha registrado en otros cultivos (Hungria et al. 2010, Díaz et al. 2011, Orona et al. 2013). Este fenómeno obedece a la efectividad simbiótica de los mecanismos que presentan algunas cepas microbianas que pueden sustituir o disminuir la adición de fertilizantes inorgánicos, con un incremento de la rentabilidad de la producción favorable y disminución de la contaminación ambiental (Adesemoye y Kloepper 2009, Nadeem et al. 2014). La práctica de inoculación microbiana en México, además de ser económica y ecológicamente segura, debe formar parte de los sistemas de agricultura sostenible.

\section{CONCLUSIONES}

Se observó un efecto positivo en el sorgo dulce derivado del empleo de las cepas microbianas HMA y BPCV, tanto en condiciones de invernadero con la variedad Dale como en campo con Cañero y Dale. En la experimentación en invernadero, la mayoría de las cepas microbianas exhibieron mejores características de planta comparadas con las plantas no inoculadas. La respuesta de Dale y Cañero a los inoculantes en campo fue variable, pero en ambas variedades siempre fue competitiva respecto a las plantas cultivadas con fertilización convencional a base de urea y fosfato monoamónico. Lo anterior se demostró con los valores promedio en ambas variedades de biomasa de tallo y contenido de azúcares — principales variables de productividad del sorgo dulce-, las cuales fueron similares entre las cepas microbianas y la fertilización convencional.

\section{AGRADECIMIENTOS}

Agradecemos el apoyo recibido de la Secretaría de Agricultura, Ganadería, Desarrollo Rural, Pesca y Alimentación (SAGARPA) de México a través del proyecto "Investigación de biofertilizantes y abonos orgánicos". A los doctores María Peña del Río, del Campo Experimental General Terán, y Armando Aguado Santacruz, del Campo Experimental Bajío, ambos del INIFAP, por proporcionarnos las cepas de HMA y BPCV, respectivamente, con las que se desarrolló esta investigación. Y a Conrado Mariano Francisco y Juan Olvera Martínez por la colaboración en los trabajos de campo.

\section{REFERENCIAS}

Adesemoye A.O. y Kloepper J.W. (2009). Plant-microbes interactions in enhanced fertilizer-use efficiency. Appl. Microbiol. Biotechnol. 85 (1), 1-12.

DOI: $10.1007 / \mathrm{s} 00253-009-2196-0$

Adesemoye A.O., Torbet H.A. y Kloepper J.W. (2009). Plant growth-promoting rhizobacteria allow reduced application rates of chemical fertilizers. Microbiol. Ecol. 58 (4), 921-929.

DOI: $10.1007 / \mathrm{s} 00248-009-9531-y$

Almodares A. y Hadi M.R. (2009). Production of bioethanol from sweet sorghum: A review. Afr. J. Agr. Res. 4 (9), 772-780.

Alvarado C.M., Díaz F.A. y Peña R.M. (2014). Productividad de tomate mediante micorriza arbuscular en agricultura protegida. Rev. Mex. Cien. Agr. 5 (3), 513-518.

Amaya C.L., Davies T.F. y Arnold A.M. (2005). Arbuscular mycorrhizal fungi, organic and inorganic controlledrelease fertilizers: Effect on growth and leachate of container-grown bush morning glory (Ipomoea carnea ssp. fistulosa) under high production temperatures. J. Amer. Soc. Hort. Sci. 130 (1), 131-139.

Askary M., Mostajeran A., Amoaghaei R. y Mostajeran M. (2009). Influence of the co-inoculation Azospirillum brasilense and Rhizobium meliloti plus 2,4-D on grain yield and N, P, K content of Triticum aestivum. Am-Eur J. Agric. Environ. Sci. 5 (3), 296-307.

Bécquer G.C., Prévost D., Juge C., Gauvin C. y Delaney S. (2012). Efecto de la inoculación con bacterias rizosféricas en dos variedades de trigo. Fase I: condiciones controladas. Rev. Mex. Cien. Agr. 3 (5), 973-984. 
Berruti A., Lumini E., Belastrini R. y Bianciotto V. (2016). Arbuscular mycorrhizal fungi as natural biofertlilizers: Let's benefit from past successes. Front. Microbiol. 6, 1559. DOI: $10.3389 /$ fmicb.2615.01559

Boucher A., Dalpe Y. y Charest C. (1999). Effect of arbuscular mycorrhizal colonization of four species of Glomus on physiological responses of maize. J. Plant Nutr. 22 (4-5), 783-797. DOI: 10.1080/01904169909365671

Calviño M. y Messing J. (2012). Sweet sorghum as a model system for bioenergy crops. Curr. Opin. Biotechnol. 23 (3), 323-329. DOI: 10.1016/jcopbio.2011.12.002

Díaz F.A., Pecina Q.V., Montes G.N., Jacques H.C. y Garza C.I. (2011). Impacto de inoculantes microbianos en sorgo cultivado bajo déficit de humedad en el suelo. En: Retos de la investigación del agua en México (S.O. Oswald, Ed.). Universidad Nacional Autónoma de México-Consejo Nacional de Ciencia y Tecnología. Ciudad de México, México, pp. 273-282.

Díaz F.A., Espinosa R.M. y Ortiz C.F.E. (2016). Promoción de biomasa y contenido de azúcares en sorgo dulce mediante abonos orgánicos y micorriza arbuscular. Rev. Int. Cont. Ambie. 32 (3), 353-360.

DOI: 10.20937/RICA.2016.32.03.09

Franco R.A., Ferrera C.R., Varela F.L., Pérez M.J. y Alarcón A. (2007). Arbuscular mycorrhizal fungi in chronically petroleum contaminated soils in Mexico and the effects of petroleum hydrocarbons on spore germination. J. Basic Microbiol. 47 (5), 378-383. DOI: $10.1002 /$ jobm. 200610293

Hamel C. y Desire G.S. (2006). Arbuscular mycorrhizal fungi in field crop production: Potential and new direction. Can. J. Plant Sci. 86: 941-950. DOI: 10.4141/ P05-099

Hungria M., Rubens C., Souza E. y Pedrosa F. (2010). Inoculation with selected strains of Azospirillum brasilense and A. lipoferum improves yield of maize and wheat in Brazil. Plant Soil 331 (1-2), 413-425. DOI: 10.1007/s11104-009-0262-0

Ilyas N. y Bano A. (2010). Azospirillum strains isolated from roots and rhizosphere soil of wheat (Triticum aestivum $\mathrm{L}$.) grown under different soil moisture conditions. Biol. Fert. Soils 46 (4), 393-406.

DOI: $10.1007 / \mathrm{s} 003374-009-0438 \mathrm{z}$

INEGI (2017). Tamaulipas: tipos de suelos [en línea]. http://www.inegi.org.mx/geo/contenidos/recnat/edafologia/default.aspx 02/02/2017.

Jetiyanon K., Fowler W.D. y Kloepper J.W. (2003). Broadspectrum protection against several pathogens by PGPR mixtures under field conditions. Plant Dis. 87 (11), 1390-1394. DOI: 10.1094/PDIS.2003.87.11.1390

Klironomos J. M. (2003). Variation in plant response to native and exotic arbuscular mycorrhizal fungi. Ecology 84 (9), 2292-2301. DOI: 10.1890/02-0413
Lopes R. V., Bespalhok F. J., Araujo L. M., Vieira R. F., Daros E. y Augusto O. R. (2012). The selection of sugarcane that display better associations with plant growth promoting rhizobacteria. J. Agr. 11 (2), 43-52. DOI: $10.3923 /$ ja.2012.43.52

Matiru V. y Dakora F. (2004). Potential use of rhizobial bacteria as promoters of plant growth for increased yield in landraces of African cereal crops. Afr. J. Biotecnol. 3 (1), 1-7. DOI: 10.5897/ajb2004.000-2002

Mau J. W. M., Houx J. H. III y Fritschi F. B. (2016). Sweet sorghum ethanol yield component response to nitrogen fertilization. Ind. Crops Prod. 84, 43-49.

DOI: $10.1016 /$ j.indcrop.2016.01.038

Montero L., Duarte C., Cun R., Cabrera J.A. y González P.J. (2010). Efectividad de biofertilizantes micorrízicos en el rendimiento de pimiento (Capsicum annuum) cultivado en diferentes condiciones de humedad del sustrato. Cultivos Trop. 31 (3), 11-14.

Montes G.N., Salinas G.J., González J.A., Loredo P.R. y Díaz P.G. (2010). Guía técnica de producción de sorgo dulce (Sorghum bicolor [L.] Moench) en Tamaulipas. Reporte Técnico No. 49. Campo Experimental Río Bravo. Instituto Nacional de Investigaciones Forestales Agrícolas y Pecuarias, Río Bravo, Tamaulipas, México, 35 pp.

Muthukumarasamy R., Govindarajan M., Vadivelu M. y Revathi G. (2006). N-fertilizer saving by the inoculation of Gluconacetobacter diazotrophicus y Herbaspirillum sp. in micropropaged sugarcane plants. Microbiol. Res. 161 (3), 238-245.

DOI: 10.1016/j.micres.2005.08.007

Nadeem S.M., Ahmad M., Zahir A., Javaid A. y Ashraf M. (2014). The role of mycorrhizae and plant growth promoting rhizobacteria (PGPR) in improving crop productivity under stressful environments. Biotechnol. Adv. 32 (2), 429-448.

DOI: 10.1016/biotechadv.2013.12.005

Orona C.F., Lozano C.M., Tucuch C.M., Grageda C.O., Medina M.S., Díaz F.A., Ruiz S. E. y Soto R.J. (2013). Response of rice cultivation to biofertilizers in Campeche, Mexico. Agr. Sci. 4, 715-720.

DOI: 10.4236/as.2013.412097

Plenchette C., Dauphin C.C., Maynard J.M. y Fortin J.A. (2005). Managing arbuscular mycorrhizal fungi in cropping systems. Can. J. Plant Sci. 85 (1), 31-40. DOI: $10.414 / \mathrm{PO} 3-159$

Plenecassagne A., Romero F.E. y López B.C. (1999). Manual de laboratorio para análisis de suelo, planta y agua. Instituto Nacional de Investigaciones Agrícolas y Pecuarias, Gómez Palacio, Durango, México. 236 p.

Prasad S. Singh A., Jain N. y Joshi H.C. (2007). Ethanol production from sweet sorghum syrup for utilization as automotive fuel in India. Energy Fuels 21 (4), 24152420. DOI: $10.1021 / \mathrm{ef060328z}$ 
Regassa H.T. y Wortmann S.C. (2014). Sweet sorghum as a bioenergy crop: Literature review. Biom. Bioe. 64, 348-355. DOI: 10.1016/j.biombioe.2014.03.052

Rego T.J., Rao V.N., Seeling B., Pardhasaradhi G. y Rao J.V. (2003). Nutrient balances-a guide to improving sorghum and groundnut-based dryland cropping systems in semi-arid tropical India. Field Crop. Res. 81 (1), 53-68. DOI: $10.1016 / \mathrm{S} 0378-4290(02) 00199-5$

Rodríguez Y.Y., Pons B.N., Fernández M.F. y Rodríguez H.P. (2004). Estudio comparativo de seis cepas de hongos micorrízicos arbusculares en su interacción con el tomate (Lycopersicun esculentum). Ecol. Aplicada 3 (1-2), 162-171.

Rolz C., de León R., Mendizábal de M.A.L., Porras V. y Cifuentes R. (2017). A multiple harvest cultivation strategy for etanol production from sweet sorghum throughout the year in tropical ecosystems. Renew. Energy 106, 103-110.

DOI: 10.1016/j.renene.2016.12.036
Sharma S., Gupta R., Dugar G. y Srivastava A. (2012). Impact of application of biofertilizers on soil structure and resident microbial community structure and function. En: Bacteria in agrobiology: Plant probiotics (D.K. Maheshwari, Ed.). Hauz Khas, Nueva Delhi, pp. 65-79.

Smith G.S. y Read D.J. (2008). Mycorrhizal symbiosis. 3a. ed. Academic Press. Londres, Inglaterra, 750 pp.

Tchabi A., Coyne D., Hountondji F., Lawouin L., Wiemken A. y Oehl F. (2010). Efficacy of indigenous arbuscular mucorrhizal fungi for promoting white yeam ( $\mathrm{Di}$ oscorea rotundata) growth in West Africa. Appl. Soil Ecol. 45 (2), 92-100.

DOI: $10.1016 /$ j.apsoil.2010.03.001

Vessey J.K. 2003. Plant growth promoting rhizobacteria as biofertilizers. Plant Soil 255 (2), 571-586. DOI: 10.1023/A.1026037216893

Wani S.P. y Lee K. (2002). Biofertilizers for sustaining cereal crops production. En: Biotechology of biofertilizers (S. Kannaiyan, Ed.). Narosa Publishing, India, pp. 50-64. 